\title{
Severe Duodenal Hemorrhage Induced by Lugol's Solution Administered for Thyroid Crisis Treatment
}

\author{
Hiroyuki Kinoshita ${ }^{1}$, Mutsuko Yasuda ${ }^{1}$, Youhei Furumoto ${ }^{1}$, Naoko Watanabe ${ }^{1}$, \\ Takao Horiuchi ${ }^{2}$, Minekazu Murayama ${ }^{1}$, Mari Kitamura ${ }^{1}$, Shingo Kaneko ${ }^{3}$, \\ Seiji Inoshita ${ }^{1}$, Yasuki Maruyama ${ }^{1}$, Matsuhiko Suenaga ${ }^{1}$, \\ Hiroshi Fujita ${ }^{4}$, Kazuhiko Fujiki ${ }^{1}$ and Fumiatsu Yakushiji ${ }^{1}$
}

\begin{abstract}
Lugol's solution is an iodinated agent used for treating thyroid crisis. It is primarily used in diagnostic tests for esophageal diseases. However, Lugol's solution can cause local mucosal injury and hemorrhage. We report, for the first time, a case of 34-year-old man who exhibited severe duodenal hemorrhage induced by Lugol's solution that was used to treat thyroid crisis. The quantity of Lugol's solution used for treating thyroid crisis is much higher than that used for mucosal disease investigation. Clinical practitioners should be aware of gastrointestinal hemorrhage when using Lugol's solution for the treatment of thyroid crisis.
\end{abstract}

Key words: thyroid crisis, gastrointestinal hemorrhage, lugol, iodine, contrast media

(Inter Med 49: 759-761, 2010)

(DOI: 10.2169/internalmedicine.49.2831)

\section{Introduction}

Thyroid crisis is a potentially fatal endocrinological emergency (1-3). Iodinated agents are essential for its treatment because they immediately retard hormone release from the thyroid gland $(2,3)$. Lugol's solution is an iodinated agent used for the treatment of thyroid crisis $(2,3)$. It is primarily used in diagnostic tests for esophageal diseases (4). To detect mucosal lesions, Lugol's solution is sprayed via endoscopic techniques. However, since Lugol's solution can cause local mucosal injury, there have been some reports on Lugol's solution-induced hemorrhage $(5,6)$. Nonetheless, gastrointestinal hemorrhage induced by Lugol's solution used for the treatment of thyroid crisis has not been reported.

Here, we report, for the first time, a case of severe duodenal hemorrhage induced by Lugol's solution that was used to treat thyroid crisis, and we discuss iodinated agents and their possible clinical applications for the treatment of thy- roid crisis.

\section{Case Report}

In December 2008, a 34-year-old man was brought to the emergency department of our hospital because he had symptoms of dyspnea, general fatigue, and nausea. The patient had no medical history of gastrointestinal disease. He was restless at the emergency department. His body temperature was $37.1^{\circ} \mathrm{C}$; his blood pressure was $130 / 97 \mathrm{mmHg}$; he had an irregular pulse rate of approximately 208 beats/min and his respiratory rate was 30 breaths/min, with approximately $84-88 \%$ oxygen saturation in room air. Radiography of the chest revealed auxocardia. An electrocardiogram showed atrial fibrillation with a heart rate of 223 beats/min. Laboratory examinations revealed the following values for various parameters: thyroid stimulating hormone (TSH), $<0.01 \mu \mathrm{U} /$ $\mathrm{mL}$ (reference range, 0.35-4.94 $\mu \mathrm{U} / \mathrm{mL}$ ); free triiodothyronine $(\mathrm{FT} 3$ ), $>30.0 \mathrm{pg} / \mathrm{mL}$ (reference range, 1.71-3.71 pg/ $\mathrm{mL}$ ); free tetraiodothyronine (FT4), $>6.00 \mathrm{ng} / \mathrm{dL}$ (reference

\footnotetext{
${ }^{1}$ Department of Internal Medicine, Tokyo Metropolitan Bokutoh Hospital, Tokyo, ${ }^{2}$ Department of Endoscopy, Tokyo Metropolitan Bokutoh Hospital, Tokyo, ${ }^{3}$ Department of Cardiology, Tokyo Metropolitan Bokutoh Hospital, Tokyo and ${ }^{4}$ Department of Transfusion Medicine, Tokyo Metropolitan Bokutoh Hospital, Tokyo

Received for publication August 31, 2009; Accepted for publication January 14, 2010

Correspondence to Dr. Hiroyuki Kinoshita, hkinoshita-tky@umin.ac.jp
} 


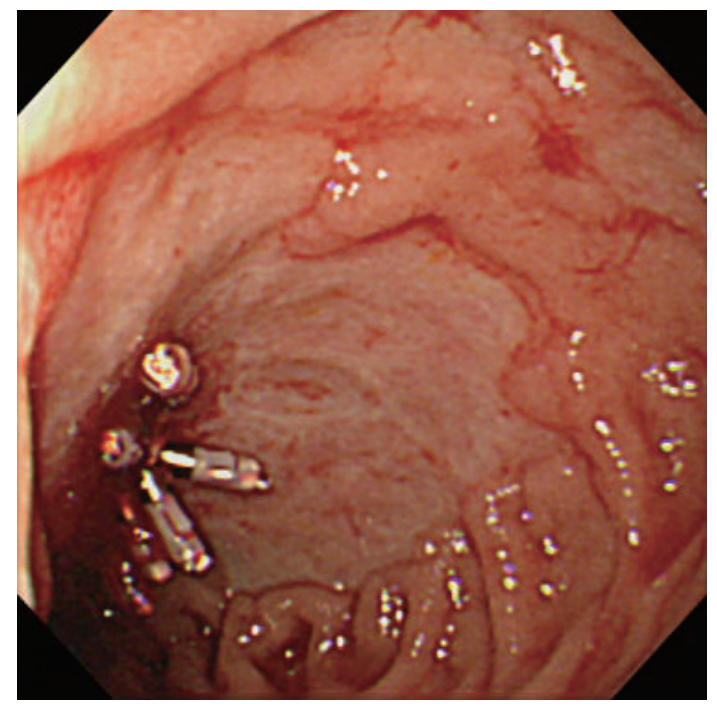

Figure 1. Endoscopy results: A geographic ulcer was observed in the second segment of the duodenum. The lesion bled easily during saline washing.

range, $0.70-1.48 \mathrm{ng} / \mathrm{dL})$; and $\mathrm{TSH}$ receptor antibody (TRAb), $63.1 \%$ (reference range, $-10.0 \%$ to $10.0 \%$ ). These conditions fulfilled the criteria of thyroid crisis by the Japan Thyroid Association (7). We therefore diagnosed this patient as thyroid crisis induced by Graves' disease.

The patient was transferred to the intensive care unit, intubated, and put on mechanical ventilation. We initiated treatment with medications such as antithyroid drugs, iodinated agents, beta-blockers, steroids (hydrocortisone, 300 $\mathrm{mg} /$ day at the first day: $200 \mathrm{mg} /$ day after the second day), and heparin (15,000 units/day). We administered 3\% Lugol's solution $32 \mathrm{~mL} /$ day (iodine intake, $960 \mathrm{mg} /$ day), via a nasogastric tube because the patient was under mechanical ventilation. Thereafter, we administered a histamine $\mathrm{H} 2$ receptor blocker to prevent gastrointestinal injury. In addition, plasmapheresis was performed with albumin solution as the replacement fluid to eliminate thyroid hormones from the plasma. Consequently, the patient's condition improved, and he was successfully extubated after five days.

We continued to administer medications, including Lugol's solution. On the fifth day after admission, the patient suddenly exhibited severe hematochezia. At that time, prothrombin time-international normalized ratio (PT-INR) was 1.27 and activated partial thromboplastin time (APTT) was $50.5 \mathrm{sec}$ (reference range, 27.0-38.0 sec). Although we administered protamine and hemostyptics and discontinued treatment with heparin and Lugol's solution, the patient went into hemorrhagic shock. We therefore transfused a total of $1,680 \mathrm{~mL}$ red blood cell solution. Gastroscopy and colonoscopy revealed duodenal hemorrhage. Endoscopy revealed the presence of a geographic ulcer in the second segment of the duodenum (Fig. 1). This lesion bled easily during saline washing. These observations were not consistent with those in the case of the conventional peptic ulcer, and the patient tested negative for Helicobacter pylori antibod- ies. We therefore diagnosed the condition as Lugol's solution-induced duodenal mucosal injury resulting in hemorrhage. After hemostasis clipping by endoscopy, we administered a proton pump inhibitor and mucosal demulcents. The hemorrhaging was thus controlled.

\section{Discussion}

Here, we report, for the first time, a case of severe duodenal hemorrhage induced by Lugol's solution, which was administered for the treatment of thyroid crisis.

Stevens et al examined various concentrations of Lugol's solution (0.5-5\%) for esophageal disease investigation, and found that the use of a higher concentration (3-5\%) may increase the risk of mucosal complications (8). In addition, Sreedharan et al recommended that a concentration of $1.5 \%$ be used (5). As per these previous reports, the patient in the present case was at a high risk of developing mucosal injury because 3\% Lugol's solution was used. A more diluted solution should have been administered to prevent local mucosal injury. Sodium thiosulfate solution is recommended for routine use after Lugol's staining in order to prevent mucosal injury, and its effectiveness has been reported (9). In the present case, sodium thiosulfate should have been administered after treatment with Lugol's solution to prevent local mucosal injury. However, sodium thiosulfate neutralizes iodine solutions, and the neutralized iodine is not absorbed by the mucosa (9). Therefore, the use of sodium thiosulfate may reduce the effectiveness of Lugol's solution for the treatment of thyroid crisis.

The typical iodide intake of people in the United States is $100-400 \mu \mathrm{g} /$ day (2). The quantity of iodine required to suppress radioactive iodine to $<2 \%$ is $>30 \mathrm{mg} /$ day (2). The quantity of iodine required for the treatment of thyroid crisis is reported to vary from 120 to $3,000 \mathrm{mg} /$ day $(2,3)$. We administered 3\% Lugol's solution at a dose of $32 \mathrm{ml} /$ day (iodine, $960 \mathrm{mg} /$ day) until just before the occurrence of hematochezia; the quantity of Lugol's solution was in the range required for the treatment of thyroid crisis; iodine, 120$3,000 \mathrm{mg} /$ day $(2,3)$. In diagnostic tests for mucosal disease, a single dose of $10-20 \mathrm{~mL}$ Lugol's solution is usually administered $(5,9)$. If $3 \%$ Lugol's solution is used, the quantity of iodine administered is $300-600 \mathrm{mg}$. Thus, the quantity of Lugol's solution used for treating thyroid crisis in the present case (iodine, $960 \mathrm{mg} /$ day) was much higher than that used in routine investigations for mucosal disease. Clinical practitioners should consider the possibility of iodinated agent-induced mucosal injury when treating thyroid crisis patients.

Iodinated contrast media are widely used for contrastenhanced computed tomography (CT). These media are also used for thyroid crisis treatment (3). Many iodinated contrast media used in Japan contain $300 \mathrm{mg} / \mathrm{mL}$ iodine, and $100 \mathrm{~mL}$ of contrast medium is usually administered for contrast-enhanced CT. Therefore, a single dose of 30,000 $\mathrm{mg}$ of iodine is intravenously administered, and this quantity 
is far greater than that used in tests for mucosal disease (300-600 $\mathrm{mg}$ at a time) and in thyroid crisis treatment (120$3,000 \mathrm{mg} /$ day). However, to the best of our knowledge, no case of contrast medium-induced gastrointestinal hemorrhage has been reported to date. We infer that the intravenous administration of iodinated contrast media is not likely to cause local mucosal injury. Thus, intravenous administration of iodinated contrast media may be considered for the treatment of thyroid crisis in terms of preventing mucosal injury. However, according to the treatment manuals of iodinated contrast media, adverse effects of their intravenous administration have been reported $1.9-6.3 \%(10,11)$. The clinical practitioners should consider the risk and benefit.

Thyroid crisis is a stressful situation, which can cause stress-induced peptic ulcers (12). In addition, steroids and iodinated agents, which cause mucosal injury, are essential for the treatment of thyroid crisis $(2,3)$. Thus, both thyroid crisis itself and its treatment measures increase the risk of mucosal injury and hemorrhage. Further, heparin is frequently administered to thyroid crisis patients because most of them exhibit atrial fibrillation, as in the present case. Furthermore, if plasmapheresis is performed with albumin solution as the replacement fluid (as in the present case), most of the coagulation factors are lost. In short, the management and treatment of thyroid crisis increases the risk of inadequate hemostasis. Further research is required to determine the best method for administering iodinated agents to treat thyroid crisis.

\section{Acknowledgement}

There is no funding source. The authors have no conflicts of interest.

\section{References}

1. Nayak B, Burman K. Thyrotoxicosis and thyroid storm. Endocrinol Metab Clin North Am 35: 663-686, 2006.

2. Kronenberg HM, Melmed S, Polonsky KS, Larsen PR. In: Williams Textbook of Endocrinology. 11th edition. Saunders Elsevier, Philadelphia, USA, 2008: 299-376.

3. Tietgens ST, Leinung MC. Thyroid storm. Med Clin North Am 79: 169-184, 1995.

4. Voegeli R. Schiller's iodine test in the diagnosis of esophageal diseases. Preliminary report. Pract Otorhinolaryngol (Basel) 28: 230-239, 1966 (in German).

5. Sreedharan A, Rembacken BJ, Rotimi O. Acute toxic gastric mucosal damage induced by Lugol's iodine spray during chromoendoscopy. Gut 54: 886-887, 2005.

6. Myung Park J, Seok Lee I, Young Kang J, et al. Acute esophageal and gastric injury: complication of Lugol's solution. Scand J Gastroenterol 42: 135-137, 2007.

7. Japan Thyroid Association. The criteria of thyroid crisis 1st edition (2008). Available at http://thyroid.umin.ac.jp/rinsyo/crise1.pdf.
Accessed February 1, 2010.

8. Stevens PD, Lightdale CJ, Green PH, Siegel LM, GarciaCarrasquillo RJ, Rotterdam H. Combined magnification endoscopy with chromoendoscopy for the evaluation of Barrett's esophagus. Gastrointest Endosc 40: 747-749, 1994.

9. Kondo H, Fukuda H, Ono H, et al. Sodium thiosulfate solution spray for relief of irritation caused by Lugol's stain in chromoendoscopy. Gastrointest Endosc 53: 199-202, 2001.

10. Instruction manual of OMNIPAQUE ${ }^{\circledR}$ (Daiichi Sankyo Co., Ltd., Tokyo, Japan). Retrieved: April 2007. Available at http://www. info.pmda.go.jp/downfiles/ph/PDF/430574_7219415G1025_2_01. pdf. Accessed February 1, 2010.

11. Instruction manual of Iopamiron ${ }^{\circledR}$ (Bayer HealthCare, Osaka, Japan). Available at http://www.bayer.co.jp/hv/products/tenpu/ipa. pdf. Accessed February 1, 2010.

12. Nikolă̌chuk LV, Grushko VS, Grushko OI. Peptic ulcer in thyrotoxicosis. Sov Med 8: 115-116, 1984 (in Russian).

(C) 2010 The Japanese Society of Internal Medicine http://www.naika.or.jp/imindex.html 